\title{
Analysis of Peroperative Lateral Femoral Cortical Burst Complication Related to ToggleLoc with ZipLoop in Anterior Cruciate Ligament Reconstruction
}

\author{
Deniz Aydın (D, Mehmet Yalçınozan (D) \\ Near East University Medical Faculty, Department of Orthopedics and Traumatology, Nicosia, Cyprus
}

ORCID iDs of the authors: D.A. 0000-0003-4|48-I42I; M.Y. 0000-0002-2772-II37.

Cite this article as: Aydın D, Yalçınozan M. Analysis of Peroperative Lateral Femoral Cortical Burst Complication Related to ToggleLoc with ZipLoop in Anterior Cruciate Ligament Reconstruction. Cyprus J Med Sci. 202I; 6(4): 279-284.

\section{BACKGROUND/AIMS}

ToggleLoc adjustable loop suspension device is a widely used implant in anterior cruciate ligament ( $A C L$ ) reconstruction, which offers several advantages. However, peroperative problems related to these devices are not clear. The objective of this study was to report and analyze the peroperative lateral femoral cortical burst complication of ToggleLoc adjustable loop suspension device in $\mathrm{ACL}$ reconstruction.

\section{MATERIALS AND METHODS}

Fifty-two patients who underwent ACL reconstruction with ToggleLoc device were reviewed for peroperative lateral femoral cortical burst complication. The relation between lateral femoral cortical burst and tunnel position on lateral femoral cortex (condylar-epicondylar) was investigated from patients' records. A case-based analysis was done. Preoperative and postoperative Lysholm scores were also noted.

\section{RESULTS}

Peroperative lateral femoral cortical burst complication was observed in four of 52 patients. In five of the 52 cases, ToggleLoc button was placed over the condylar region, and four of the cases with condylar button placement had the complication. The mean preoperative and postoperative Lysholm scores were 37.98 (min: 26, max: 62) and 91.73 (min: 81, max: 100), respectively.

\section{CONCLUSION}

ToggleLoc button structure and distal loading of tensioning sutures cause increased risk of lateral femoral cortical burst during $\mathrm{ACL}$ reconstruction. As a precaution, condylar placement of the femoral tunnel lateral cortical aperture should be avoided.

Keywords: Anterior cruciate ligament, femoral fixation, suspension device, adjustable loop

\section{INTRODUCTION}

Adjustable loop cortical suspension devices have become increasingly popular in femoral fixation of quadrupled hamstring grafts in anterior cruciate ligament (ACL) reconstruction. ToggleLoc (ToggleLoc ${ }^{T M}$ Device with ZipLoop ${ }^{R}$ Technology) is an widely used adjustable cortical suspensory device in ACL reconstruction. These devices are aimed to provide several advantages: single implant with adjustable technique fits all femoral socket-tunnel measurements, extraspace is not necessary in femoral socket for flipping button over cortex so longer graft placed in femoral socket, decreased distance between button and graft may reduce the bungee effect, and also re-tensioning on femoral side is possible after tibial fixation. ${ }^{1,2}$ On the other hand, some concerns are raised about these devices. As the similar complications like fixed loop devices could be expected, another problem of loosening in adjustable loop is questioned in literature. ${ }^{2,3}$ In a technical report about ToggleLoc, the complication of lateral femoral cortical breakage was mentioned in a 45-years-old male, and it is suggested to place suspensory button proximally to avoid thin and weak distal metaphyseal area, especially in older patients. ${ }^{4}$

Lateral femoral cortical burst and metaphyseal escape of suspensory button are unusual complications in ACL reconstruction. In this paper, we report this peroperative complication related to ToggleLoc with ZipLoop device and 


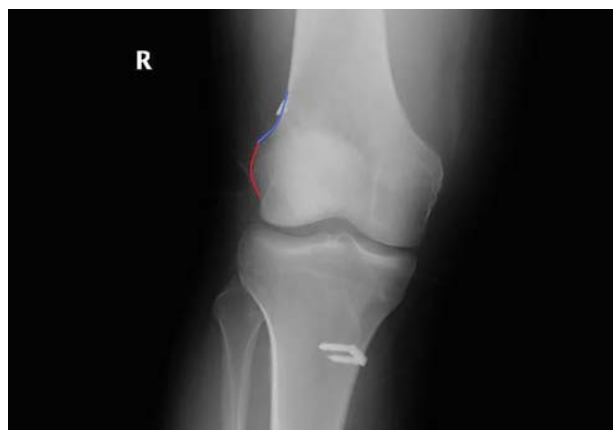

Figure I. Anteroposterior knee X-ray. Condylar region is marked with red and epicondylar region with blue.

hypothesized that it is more common with condylar placement of the suspensory button. We discuss the mechanism of this complication to present the reasons and technical pitfalls for prevention.

\section{MATERIALS AND METHODS}

Hospital records of 52 patients who underwent ACL reconstruction with ToggleLoc between January 2016 and March 2017 in a single institution were evaluated retrospectively. Cases with anatomic single bundle reconstruction through accessory medial portal with quadrupled hamstring autograft were included in the study. ACL reconstructions with transtibial femoral tunnel preparation, femoral fixation technique other than ToggleLoc device, and grafts other than quadrupled hamstring autograft were excluded from the study. All the surgeries were done by the two surgeons with experience more than 5 years. Age, sex, side, injury mechanism, and associated injuries were recorded. Tunnel position on lateral femoral cortex (condylar-epicondylar) was analyzed by using postoperative anteroposterior knee X-rays. Condylar and epicondylar regions were shown in Figure I. Distance between lateral cortex to graft (femoral tunnel length - femoral socket depth difference) and graft size was investigated with operation notes. Preoperative and postoperative Lysholm scores were recorded for all patients.

Operation notes and videos were reviewed to document cases with peroperative complications. Four cases were reached. A

\section{Main Points}

- Femoral fixation of quadrupled hamstring autograft with ToggleLoc Ziploop system in anterior cruciate ligament reconstruction provides satisfactory results with good postoperative Lysholm scores.

- Lateral femoral cortical burst during ACL reconstruction with ToggleLoc button is not a rare peroperative complication.

- ToggleLoc button structure and distal loading of tensioning sutures cause increased risk of lateral femoral cortical burst during $\mathrm{ACL}$ reconstruction.

- As a precaution, surgeons should avoid condylar placement of the femoral tunnel lateral cortical aperture and femoral tunnel-hamstring graft size mismatch. case-based analysis of peroperative complications for possible reasons and salvage methods was done.

This study was approved by Near East University Ethics Review Board on 20.04.2017 with project number YDU/2017/ 46-395. All the patients gave written informed consent for the publication of their individual data.

\section{Surgical Technique}

Patients were operated in supine position under tourniquet with legs swinged from operating table. Diagnostic arthroscopy was done to correlate $A C L$ rupture and evaluate associated intra-articular injuries. Hamstring tendons were harvested, prepared, and placed to ToggleLoc device in the usual manner. Accessory medial portal was created to prepare femoral tunnel. Guide pin was placed through the accessory portal over the anatomic femoral footprints of $A C L$, and the knee was flexed to $100-120^{\circ}$ while advancing the guide pin anterolaterally. The knee hyperflexion was provided by combined flexion of hip in some cases, and the technique provides a more horizontal and anterior directed femoral tunnel to avoid posterior wall blow-out. The knee was kept in the same position to create button tunnel with $4.5 \mathrm{~mm}$ drill and the femoral socket with drill size equal to prepared graft. The length of button tunnel was measured. Femoral socket was created $25 \mathrm{~mm}$ depth, routinely. Tibial tunnel was drilled in the same size with the graft over a guide wire placed from medial tibial cortex to the center of ACL tibial footprint. Length of the femoral button tunnel was marked on ToggleLoc loops, depth of the femoral socket was marked on quadrupled graft, and tensioning sutures were signed to distinguish from suspensory loops. Passing suture attached to the one side of ToggleLoc button was moved from tibial tunnel to femoral tunnel and passed through anterolateral surface of leg. ToggleLoc button was brought to the femoral lateral cortex with the help of passing suture. It was cautioned to pull ToggleLoc button smooth surface laterally. Passing suture was pulled medially to flip ToggleLoc button, while the marks on loop reach to the femoral tunnel intra-articular aperture. The graft was moderately tensioned distally during all procedure. The presigned tensioning sutures were passed to the medial portal. The graft was pulled from the tibial tunnel to the femoral socket by the force applied distally to the tensioning sutures. Once the marked line over the graft reached to the femoral tunnel intra-articular aperture, tensioning was stopped. The knee was cycled 10 times with manual loading for pretensioning, and then the system was retensioned for possible displacement. Tibial fixation was done in $20^{\circ}$ knee flexion under manual tension. The tibial side was fixed with tunnel sized diameter and $20 \mathrm{~mm}$ length bioabsorbable screw, which was reinforced with staple. Graft tension, knee stability, and range of motion without impingement were checked before ending the operation.

\section{RESULTS}

Fifty-two patients were evaluated in the study. Four were female and 48 were male. The age of patients ranged from 18 to 44 (mean 28.7, median 28). Measured graft sizes and corresponding tunnel diameters were $7 \mathrm{~mm}$ in two, $8 \mathrm{~mm}$ in 46 , and $9 \mathrm{~mm}$ in four patients. Femoral socket depth was standardized to $25 \mathrm{~mm}$. Minimum and maximum femoral tunnel lengths created with $4.5 \mathrm{~mm}$ drill were 37 and $52 \mathrm{~mm}$, respectively. So, the minimum distance between ToggleLoc button and graft was $12 \mathrm{~mm}$, and maximum distance was $27 \mathrm{~mm}$ (mean I8.I, median 


\begin{tabular}{|c|c|c|c|c|}
\hline & Case I & Case 2 & Case 3 & Case 4 \\
\hline Age & 44 & 23 & 28 & 20 \\
\hline Sex & Male & Male & Male & Male \\
\hline Associated injuries & $\begin{array}{l}\text { Patellofemoral arthritis } \\
\text { Degenerative medial meniscal tear }\end{array}$ & None & Lateral meniscus posterior horn tear & None \\
\hline Tunnel placement & Condylar & Condylar & Condylar & Condylar \\
\hline Graft size & $8 \mathrm{~mm}$ & $8 \mathrm{~mm}$ & $8 \mathrm{~mm}$ & $8 \mathrm{~mm}$ \\
\hline Preoperative Lysholm score & 37 & 34 & $4 \mid$ & 49 \\
\hline Postoperative Lysholm score & Not reported & 90 & 94 & 86 \\
\hline
\end{tabular}

18). In 47 of the cases, femoral button placement was over the epicondylar region, and in five of them, it was condylar.

Lateral cortical burst with metaphyseal migration of the ToggleLoc button was observed in four patients, and no other major peroperative complication was observed. Four of the cases with condylar placement of the button had the lateral cortical burst complication. Demographic characteristics and case analysis of patients with peroperative complication were given in Table I.

The mean preoperative Lysholm score was 37.98 (min: 26, max: 62). Postoperative Lysholm score was available in 42 of the patients with a mean follow-up period of 25 months (1439 months). Four of the patients with peroperative complication were not included in these 42 patients. The mean postoperative Lysholm score was 91.73 (min: 81, max: 100). Reconstruction failure was observed in two of the 42 patients.

\section{Case I}

A 44 year-old-male was injured II months before operation. Injury mechanism was twisting of the knee while landing from I $\mathrm{m}$ height. Mild patellofemoral arthritis and degenerative posterior medial meniscal tear were associated. ACL reconstruction was done. was done. Graft was measured as $8 \mathrm{~mm}$, $4.5 \mathrm{~mm}$ femoral button tunnel was $42 \mathrm{~mm}$, and $25 \mathrm{~mm}$ femoral socket was created. Lateral cortical burst was distinguished at the end of surgery after tibial fixation while moving the knee in full range of motion for testing. Tibial fixation was removed, and graft with ToggleLoc system was taken out. It was observed that there is no distance between graft and loop. As it was assumed that button was not seated over the lateral cortex, a new device was used for suspension and checked with fluoroscopy for appropriate seating of device over the lateral cortex. However, button was migrated again even with initial loading on tensioning sutures. Lateral cortical burst was diagnosed. Lateral incision was used to approach lateral cortex, and screw washer was placed between ToggleLoc button and lateral cortex. The graft was pulled into planned position and fixed. Postoperative X-rays showed condylar placement of button. Standard rehabilitation was applied to the patient with no complication on the third month, but he missed the later follow-ups, so his clinical and radiological results are unknown.

\section{Case 2}

A 23 year-old-male was injured during football game. No associated intra-articular lesion was existed. Reconstruction was done II months after initial injury for recurrent giving way com- plaints of the knee. Graft diameter was $8 \mathrm{~mm}$, femoral tunnel was $40 \mathrm{~mm}$, and femoral socket was $25 \mathrm{~mm}$. Lateral cortical burst was not understood peroperatively, as the knee was stable, and graft was tensioned during peroperative evaluation. However, postoperative x-rays showed metaphyseal migration of button (Figure 2a). Lateral aperture of femoral tunnel was on condylar region. He was out of complaint in his 2 year follow-up with stable knee and no further displacement of button (Figure 2b). His preoperative and postoperative Lysholm scores were 34 and 90, respectively.

\section{Case 3}

A 28-year-old male reported that he fell while he is running on an uneven surface. ACL rupture and lateral meniscus posterior horn tear were diagnosed. He was operated Imonth after injury. A $8 \mathrm{~mm}$ diameter hamstring graft was used with $39 \mathrm{~mm}$ femoral tunnel and $25 \mathrm{~mm}$ femoral socket. Lateral cortical burst was diagnosed during graft passage from tibial tunnel to femoral tunnel. Sudden loosening of the tensioning sutures with a crack voice was observed. ToggleLoc system was replaced by Endobutton fixed loop device. Postoperative $x$-rays revealed condylar placement of button. He was out of complication in his 2-year follow-up with return to sports. His preoperative and postoperative Lysholm scores were $4 \mathrm{I}$ and 94 , respectively.

\section{Case 4}

A 20-year-old male was injured 6 months before operation during a football game. No associated intra-articular lesion was existed. Graft diameter, femoral tunnel, and socket lengths were 8,37 , and $25 \mathrm{~mm}$, respectively. Lateral cortical burst was diagnosed during graft passage through the tibial tunnel. Sudden loosening of the tensioning sutures with a crack voice was observed. The ToggleLoc system was replaced by the Endobutton fixed loop device. However, the graft was again stucked in tibial tunnel. The graft diameter was measured again. As the tunnels were $8 \mathrm{~mm}$, the graft was hardly passed from that diameter on the measurement guide. Tunnel diameters were increased to $8.5 \mathrm{~mm}$, and later, the graft passage and endobutton fixation were easily provided. Postoperative radiographies showed that the button was placed distally over the condylar region cortex. No complaint or radiological problem was reported in his 15 month follow-up. His preoperative and postoperative Lysholm scores were 49 and 86, respectively.

\section{DISCUSSION}

In this study, it was found that lateral cortical burst and metaphyseal escape of button are not rare peroperative 


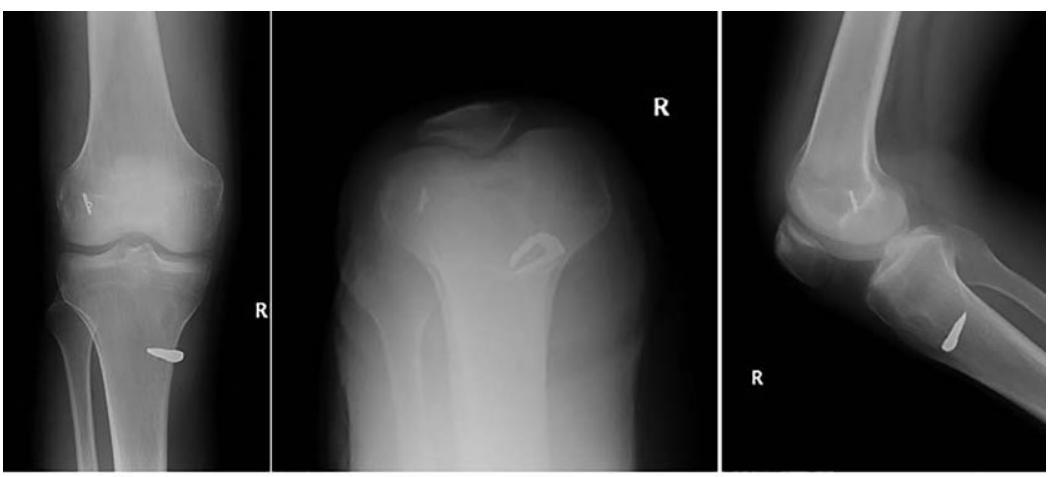

(a)

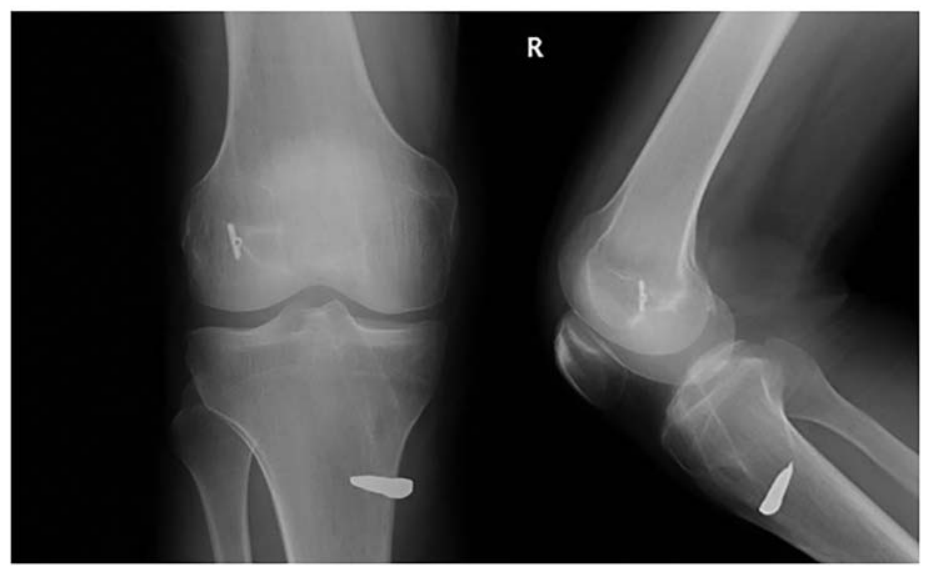

(b)

Figure 2. (a) Early postoperative knee X-rays of the case 2. It shows metaphyseal migration of ToggleLoc button. (b) Knee X-rays show that the button is not further displaced during the 2-year follow-up.

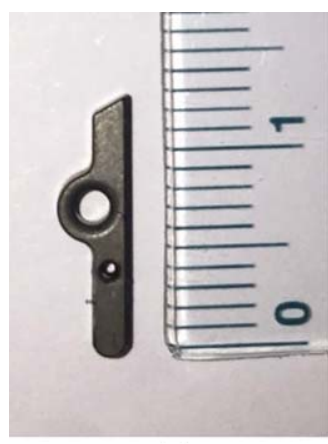

(a)

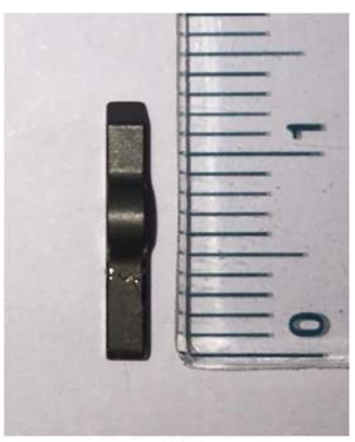

(b)
Figure 3. (a) Lateral picture of ToggleLoc button. (b) Undersurface picture of ToggleLoc button. It has an asymmetric structure, and the undersurface of the button has a noncentered ring for adjustable loop.

complications of ToggleLoc device in ACL reconstruction. Surgeons are not familiar with this problem as it is not usual by using other suspensory fixation devices.

ToggleLoc device has some unique properties, but these may cause undesired problems. The width of the ToggleLoc button is narrower than the other suspensory devices. The button has an asymmetric structure, and the undersurface of the button has a ring for adjustable loop, which is not centered (Figure 3). In other suspensory devices (Smith $\delta$ Nephew Endobutton $\mathrm{CL}$, Arthrex RetroButton, Linvatec XO Button), there are two holes on a symmetric button structure. ${ }^{5}$ Vertically applied forces to the button should be evenly spread over the lateral femoral cortical surface. Unevenly affecting forces between button and cortex will increase pressure on bone with same load acting on a decreased area. An object with symmetrically centered multiple acting points like two holed suspensory devices is more balanced. ToggleLoc button has an uneven load distribution with one acting point, which is not centered. Figure 4 shows that ToggleLoc button arms are unevenly seated on sides of drill hole. In theory, ToggleLoc button structure could contribute lateral femoral cortical failure with increased forces on a smaller area cause of narrower width, asymmetric shape, and single noncentered suspension point. However, in a biomechanical study, cortical failure of fresh frozen porcine femur bone was reported in only $10 \%$ of constructs, which is same for Endobutton and XO Button, and ultimate failure load is also close to them. ${ }^{6}$

The zipping system of ToggleLoc adjustable loop is also unique. The tensioning sutures are pulled distally to place the graft into the femoral tunnel. ToggleLoc button acts like a pulley system. The applied force to the tensioning sutures is equal to the force created on the adjustable loop sutures. The sum of these two forces or twice the force we applied to the 


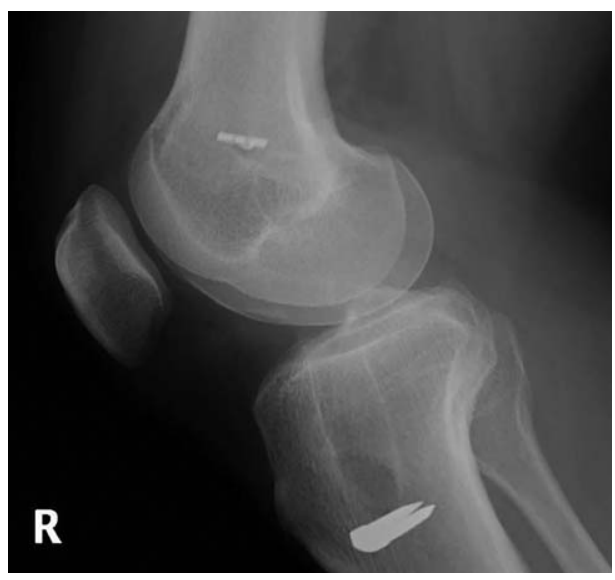

Figure 4. ToggleLoc button arms are unevenly seated on sides of drill hole.

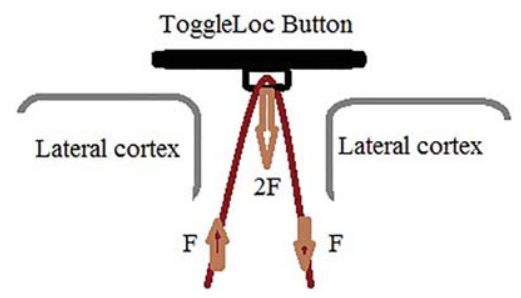

Figure 5. The force acting over the lateral femoral cortex by ToggleLoc button is twice the force applied to the tensioning sutures as the button works like a pulley system.

tensioning sutures is the load over the ToggleLoc button. So, the force acting over the lateral femoral cortex by ToggleLoc button is twice the force necessary to bring the graft from tibial tunnel to the femoral tunnel (Figure 5). In anatomic ACL reconstruction, the axis of these two tunnels is different. As a result, the necessary force to pull the graft from tunnels is increased. Any obstacle in graft passage will increase the load on tensioning sutures. Unprepared tibial tunnel intra-articular aperture with $A C L$ remnants or failure to advance drill for appropriate preparation of articular orifice may cause stucking of graft during passage. Also, graft-tunnel size mismatch (a larger graft in a smaller tunnel) will cause big forces over lateral femoral cortex. Measurement mistake or swelling of the graft (while preparing the tunnels, the prepared graft is kept in a saline soaked gauze to prevent from drying) could be the reason for graft-tunnel size mismatch. Graft-tunnel size mismatch is a very serious problem, and it is irreversible if the graft is stucked in the tibial tunnel. In this case, all the system is squeezed, and there is no room for flipping the button reversely. Excessive forces are applied in this situation, which may result with passage of the graft or breakage of the lateral femoral cortex. Graft-tunnel mismatch was observed in I of our complicated cases. We believe that graft swelling after measurement was the reason for mismatch. The problem was solved by enlarging the tunnels' diameter, and Endobutton $\mathrm{CL}$ was used for femoral side fixation.

In our study, all the four cases with peroperative complication had the metaphyseal placement of the ToggleLoc button. Cer- tain loads are applied over the lateral femoral cortex during implantation of the graft. So, we suggest that the ToggleLoc button should be placed on a stronger area as far as possible. As the diaphyseal cortex is much more stronger than metaphyseal area, more proximal placement of femoral tunnel external aperture provides stronger support for button. Vertical tunnels are necessary for higher placement of femoral tunnel external aperture. Independent femoral tunnel drilling from an accessory medial portal cause more horizontal tunnel placement than transtibial femoral tunnel drilling. ${ }^{7}$ In anatomic reconstructions through the accessory medial portal, the angle between drill and lateral notch wall could be decreased to provide a higher femoral tunnel external aperture placement. However, decreased angle between drill and lateral notch wall could cause cartilage damage on posterior articular surface of lateral femoral condyle and posterior wall blowout. ${ }^{8}$

In the case of lateral cortical burst with ToggleLoc, many salvage procedures are possible. However, revision from the same tunnel with a new ToggleLoc device is useless. In our first complication, we did not recognize the problem in the beginning and tried on revision with a new ToggleLoc device. As it was unsuccessful, we used lateral approach to define the problem. We used screw washer between bone and button cause of lateral cortical breakage. Although no problem was observed in the patient's 3-month follow-up, this kind of fixation is not advisable cause of button slippage risk. In the next two complications, we met peroperatively, we used Endobutton $\mathrm{CL}$ fixation as it covers a wider surface over the lateral femoral cortex. In a study, Hammond et al. reported that three femoral cortical suspensory fixation devices (Linvatec XO Button, Arthrex RetroButton and Smith $\delta$ Nephew Endobutton $C L$ ) provide satisfactory fixation strength even in the case of lateral femoral cortical breach with $8 \mathrm{~mm}$ or smaller diameter drill. ${ }^{5}$

\section{LIMITATIONS}

There are several limitations in this study. It presents small number of patient, and it reflects the experiences of a single institution. So, this study is unable to estimate the true prevalence of the peroperative lateral cortical burst complication related to ToggleLoc system. Another week point is the lackness of a biomechanical study to compare the ultimate failure load of condylar and epicondylar regions with ToggleLoc button fixation. Statistical analysis was not used as the number of complicated cases, and cases with condylar button placement are very low.

\section{CONCLUSION}

ToggleLoc button structure and distal loading of tensioning sutures cause increased risk of lateral cortical burst during $A C L$ reconstruction. As a precaution, condylar placement of the femoral tunnel lateral cortical aperture should be avoided. Also, graft size should be remeasured just before implantation, and tunnels should be well prepared to prevent catching of the graft in the tunnels. Surgeon should be ready for alternative fixation methods in the case of lateral cortical burst.

Ethics Committee Approval: Ethical committee approval was received from the Near East University Ethics Review Board (YDU/2017/46395)(20.04.2017).

Informed Consent: Written informed consent was obtained from all participants who participated in this study. 
Peer-review: Externally peer-reviewed.

Author Contributions: Concept - D.A., M.Y.; Design - D.A., M.Y.; Supervision - M.Y.; Resources - M.Y.; Materials - D.A.; Data Collection and/or Processing - D.A., M.Y.; Analysis and/or Interpretation - D.A.; Literature Search - M.Y; Writing Manuscript - D.A., M.Y.; Critical Review - D.A.

Conflict of Interest: The authors have no conflicts of interest to declare.

Financial Disclosure: The authors declared that this study has received no financial support.

\section{REFERENCES}

I. Nye DD, Mitchell WR, Liu W, Ostrander RV. Biomechanical comparison of fixed-loop and adjustable-loop cortical suspensory devices for metaphyseal femoral-sided soft tissue graft fixation in anatomic anterior cruciate ligament reconstruction using a porcine model. Arthroscopy. 2017;33(6):1225-1232. [CrossRef]

2. Choi $\mathrm{NH}$, Yang BS, Victoroff BN. Clinical and radiological outcomes after hamstring anterior cruciate ligament reconstructions: Comparison between fixed-loop and adjustable-loop cortical suspension devices. Am J Sports Med. 2017;45(4):826-831. [CrossRef]

3. Houck DA, Kraeutler MJ, McCarty EC, Bravman JT. Fixed-versus adjustable-loop femoral cortical suspension devices for anterior cruciate ligament reconstruction: A systematic review and metaanalysis of biomechanical studies. Orthop J Sports Med. 2018;6(I0):2325967|I8801762.

4. Kubota M, Ohno R, Sato T, Yamada D, Fukusato S, Kaneko K. Pearls and pitfalls of the ToggleLoc with ZipLoop for anterior cruciate ligament reconstruction. Arthrosc Tech. 2018;7(8):e863-e867. [CrossRef]

5. Hammond KE, Dierckman BD, Potini VC, Xerogeanes JW, Labib SA, Hutton WC. Lateral femoral cortical breach during anterior cruciate ligament reconstruction: A biomechanical analysis. Arthroscopy. 2012;28(3):365-37I. [CrossRef]

6. Petre BM, Smith SD, Jansson KS, et al. Femoral cortical suspension devices for soft tissue anterior cruciate ligament reconstruction: $\mathrm{A}$ comparative biomechanical study. Am J Sports Med. 2013;4I(2):416-422. [CrossRef]

7. Clockaerts $S$, Van Haver A, Verhaegen J, et al. Transportal femoral drilling creates more horizontal $A C L$ graft orientation compared to transtibial drilling: A 3D CT imaging study. Knee. 2016;23(3):412-419. [CrossRef]

8. Zantop T, Haase AK, Fu FH, Petersen W. Potential risk of cartilage damage in double bundle $A C L$ reconstruction: Impact of knee flexion angle and portal location on the femoral PL bundle tunnel. Arch Orthop Trauma Surg. 2008;128:509-513. [CrossRef] 